\title{
ARTEMIS, THE NEW ${ }^{14}$ C AMS AT LMC14 IN SACLAY, FRANCE
}

\author{
E Cottereau ${ }^{1,2} \bullet \mathrm{M} \mathrm{Arnold}^{3} \bullet \mathrm{C} \mathrm{Moreau}^{1} \bullet \mathrm{D} \mathrm{Baqué}^{1} \bullet \mathrm{D} \mathrm{Bavay}^{1} \bullet \mathrm{I}^{\text {C Caffy }}{ }^{1} \bullet \mathrm{C} \mathrm{Comby}^{1} \bullet$ \\ J-P Dumoulin ${ }^{1} \bullet$ S Hain ${ }^{1} \bullet$ M Perron ${ }^{1} \bullet \mathrm{J} \mathrm{Salomon}^{4} \bullet$ V Setti $^{1}$
}

\begin{abstract}
The new facility Artemis was installed in 2003 in Saclay, France. This 3MV NEC Pelletron is dedicated to high-precision radiocarbon measurements for French ${ }^{14} \mathrm{C}$ laboratories. We will present information on Artemis along with our sample preparation methods. Results from measurements on some intercalibration samples will be given along with the values of measured blanks. Finally, we report on some problems we have encountered when measuring sputter cathodes with high $\mathrm{CH}^{-}$outputs.
\end{abstract}

\section{INTRODUCTION}

Since the early 1980s, a multi-mass accelerator mass spectrometry (AMS) system (Arnold et al. 1987) has been operating in Gif sur Yvette, France. Since then, the demand for radiocarbon measurements has been continuously growing in France. In the late 1990s, the demand from research laboratories from different organizations (CNRS, CEA, IRD, IRSN and Ministère de la Culture et de la Communication) was estimated to be about 4500 a year. It was thus decided to purchase a dedicated ${ }^{14} \mathrm{C}$ AMS system. The Laboratoire de Mesure du Carbone 14 (LMC14) was set up in Saclay around Artemis, an AMS system based on a 3MV Pelletron purchased from National Electrostatics Corporation (NEC; Middleton, Wisconsin, USA) and installed early 2003. We will present the details of Artemis along with our sample preparation methods. Results from measurements on some intercalibration samples will be given along with the values of measured blanks. We will also report on some problems we have run into in measuring sputter cathodes with high $\mathrm{CH}^{-}$output.

\section{DESCRIPTION OF ARTEMIS}

Artemis (Figure 1) is equipped with 2 MC-SNICS cesium sputter ion sources, one capable of handling 40 samples and the other 134 samples, in order to minimize downtime. This allows us to install a gas ion source in the future. Each cycle includes the injection of ${ }^{12} \mathrm{C}$ during $0.170 \mathrm{~ms}$, of ${ }^{13} \mathrm{C}$ during $1.02 \mathrm{~ms}$, and finally of ${ }^{14} \mathrm{C}$ during $100 \mathrm{~ms}$. The cesium beam stays on the cathode during 2000 cycles before the next cathode moves in front of the Cs beam.

The target wheel in the 134-sample ion source has been moved $2 \mathrm{~mm}$ back from the factory setting so that the distance between the cesium focus electrode and the target increased. The ${ }^{12} \mathrm{C}$ current on the low-energy side increased from 25 to $50 \mu \mathrm{A}$. The transmission, defined as ${ }^{12} \mathrm{C}^{3+/ 12} \mathrm{C}^{-}$, is routinely $50 \%$ for both sources; it includes the stripping yield to the $3+$ charge state.

A $45^{\circ}$ electrostatic spherical analyzer directs the beam to the $90^{\circ}$ magnetic analyzer. ${ }^{12} \mathrm{C}^{-}$and ${ }^{13} \mathrm{C}^{-}$ beams are measured in an offset Faraday cup. The different isotopes are sent sequentially through the accelerator, and the negative ions are stripped using argon. The terminal is operated at $2.6 \mathrm{MV}$. A $110^{\circ}$ magnetic analyzer sends the high-energy beam into the ${ }^{14} \mathrm{C}$ line and through a $20^{\circ}$ electrostatic cylindrical analyzer (ECA). ${ }^{12} \mathrm{C}^{3+}$ and ${ }^{13} \mathrm{C}^{3+}$ are measured in 2 offset Faraday cups at the exit

\footnotetext{
${ }^{1}$ LMC14, Bât 450 porte 4E, CEA Saclay, 91191 Gif sur Yvette, France.

${ }^{2}$ Corresponding author. Email: evelyne.cottereau@cea.fr.

${ }^{3}$ CEREGE, BP80, 13545 Aix en Provence Cedex 04, France.

${ }^{4}$ C2RMF, Palais du Louvre 75001 Paris, France.
} 


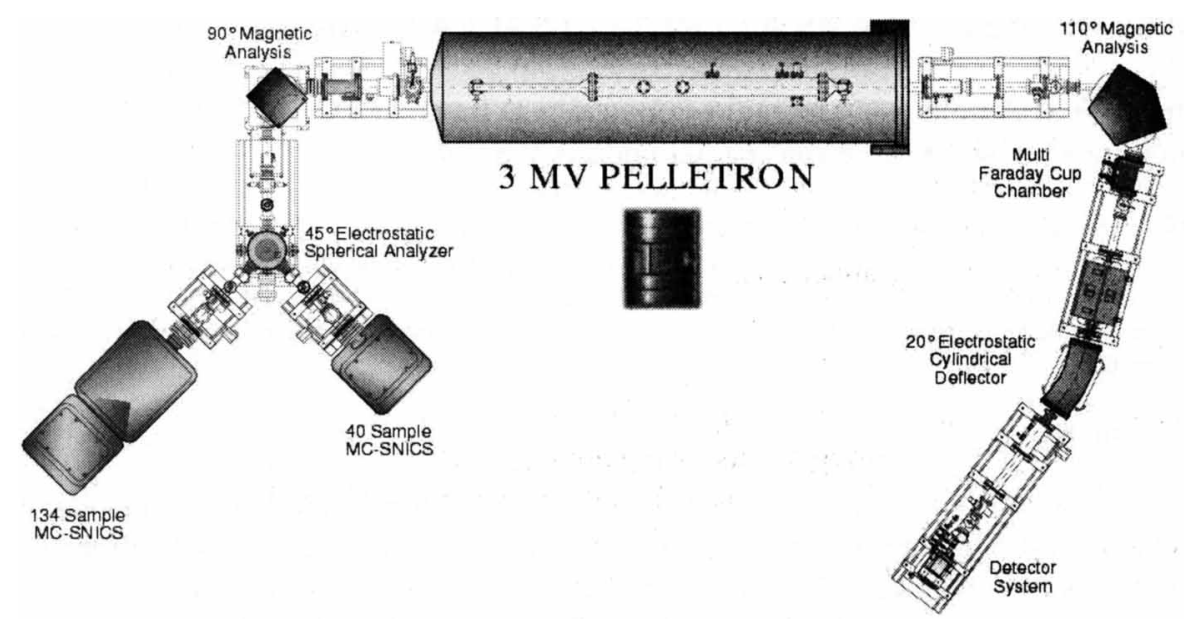

Figure 1 Artemis, 3MV Pelletron AMS system

of the magnet. The ions are then counted in an ionization chamber detector. The accelerator runs unattended after tuning.

Typical running conditions for the sources are as follows:

- Cathode voltage: $-6.5 \mathrm{kV}$

- Extractor voltage: $18 \mathrm{kV}$

- Einzel lens voltage: $0 \mathrm{kV}$

- High-voltage bias: $-45 \mathrm{kV}$

- Ionizer: 180-200 W

134-sample source:

- Cesium focus voltage: $-1.6 \mathrm{kV}$

- Cs line heater: $150 \mathrm{~W}$

- Cs oven temperature: $240{ }^{\circ} \mathrm{C}$

40-sample source:

- Cesium focus voltage: $-1.4 \mathrm{kV}$

- Cs line heater: $25 \mathrm{~W}$

- Cs oven temperature: $190^{\circ} \mathrm{C}$

We use "abc," NEC's standard data acquisition software to process the raw data and calculate the $\delta^{13} \mathrm{C}$, the average ${ }^{14} \mathrm{C} /{ }^{12} \mathrm{C}$ ratio, and the average ${ }^{13} \mathrm{C}^{3+} / 12 \mathrm{C}^{3+}$ ratio. These data are then transferred to an in-house database to calculate the age and the associated error.

\section{SAMPLE PREPARATION}

Carbonate samples dated in our laboratory include mollusk shells, foraminifera shells, and corals. Pretreatment of the samples usually starts with ultrasonic cleaning, sand blasting, and abrasion. The samples are then partially dissolved with $10^{-2} \mathrm{M} \mathrm{HNO}_{3}$. The samples along with $1 \mathrm{~cm}^{3}$ of pure $\mathrm{H}_{3} \mathrm{PO}_{4}$ acid are placed on a semi-automatic rig (Hatté et al. 2003) that enables us to produce $5 \mathrm{CO}_{2}$ samples per day. 
Organic samples dated in our laboratory include wood, charcoal, soils, plants, peat, and sediments. Pretreatment depends on the samples, but usually the samples are pretreated using the classical acidalkali-acid (AAA) method. After pretreatment, the sample is dried and placed in a quartz tube along with an excess of $\mathrm{CuO}$ and some silver wire. Up to 5 tubes can be placed on a semi-automatic rig (Tisnérat-Laborde et al. 2001). The sample is burned at $850^{\circ} \mathrm{C}$ for $5 \mathrm{hr}$, and the $\mathrm{CO}_{2}$ produced is then automatically collected in vials. It takes 2 days from installation of the tubes to collection of the $\mathrm{CO}_{2}$. Another rig is currently under construction that will allow us to collect $7 \mathrm{CO}_{2}$ samples a day.

\section{GRAPHITE TARGET PREPARATION}

Two groups of $6 \mathrm{CO}_{2}$ samples can be graphitized automatically according to the reaction described by Vogel et al. (1984). Each group has its own pumping system composed of a turbomolecular and a primary pump. The $\mathrm{CO}_{2}$ samples are placed on the vacuum line (\#1 in Figure 2). The catalyst Fe powder (average particle size $10 \mu \mathrm{m}$ ) is placed in a quartz tube (\#2 in Figure 2); the weight of the Fe powder is 3 times the weight of carbon. The volume of the reduction line is approximately $18 \mathrm{~cm}^{3}$. The system is pumped overnight and outgassed at $100{ }^{\circ} \mathrm{C}$. A multi-temperature device allows us to control the temperature of the cold finger (\#3 in Figure 2) to suit the different steps of the reduction with only liquid nitrogen as a cryogenic fluid:

- $-150{ }^{\circ} \mathrm{C}$ to trap $\mathrm{CO}_{2}$;

- $-70{ }^{\circ} \mathrm{C}$ to trap $\mathrm{H}_{2} \mathrm{O}$ during the reaction;

- $25^{\circ} \mathrm{C}$ to expand $\mathrm{CO}_{2}$ and measure the pressure in the calibrated volume.

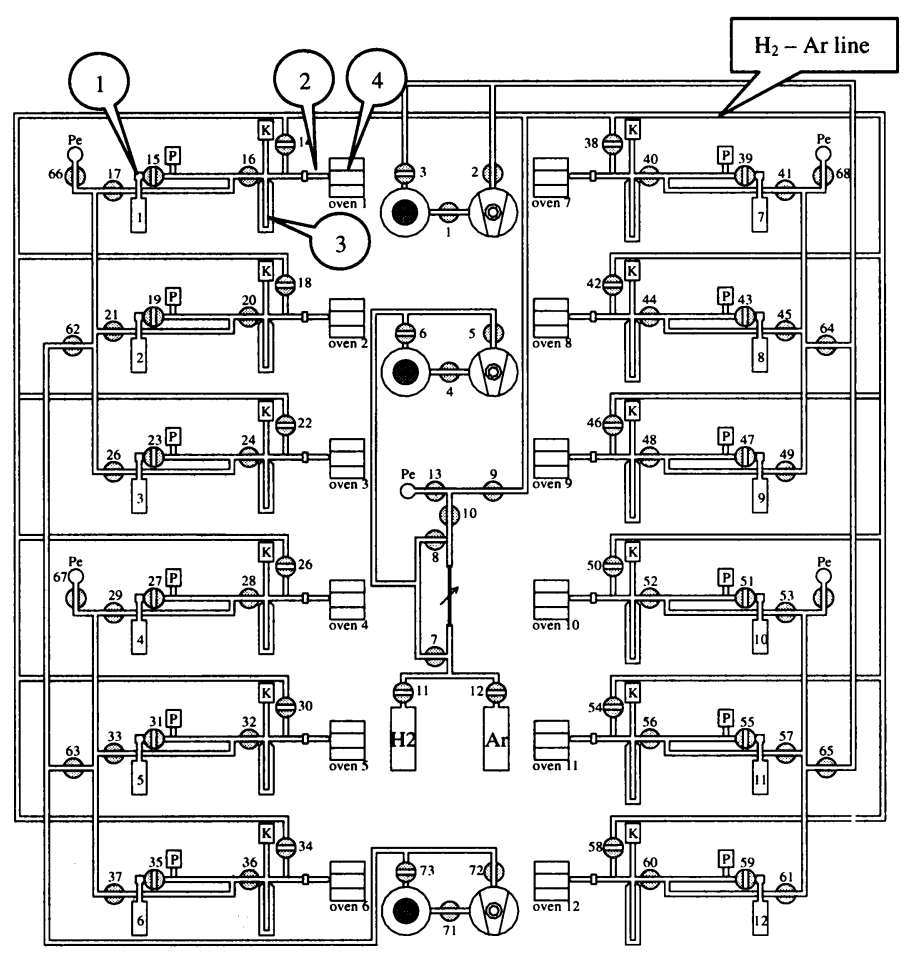

Figure 2 Schematics of the semi-automated graphitization system: 1 - vacuum line; 2 - reduction tube; 3 - cold finger (see text for details). 
The entire system is monitored by a PC computer controlled by software written in Delphi (Turbo Pascal Object) that collects the different parameters (pressure, temperature). The valves are either electropneumatically or vacuum actuated. $\mathrm{H}_{2}$ is introduced at a pressure a little over twice the pressure of $\mathrm{CO}_{2}$ in the calibrated volume at $25^{\circ} \mathrm{C}$. The oven is set to $575{ }^{\circ} \mathrm{C}$ after the $\mathrm{H}_{2}$ is introduced and the temperature of the finger is set to $-70^{\circ} \mathrm{C}$.

The reduction lasts usually $3-4 \mathrm{hr}$. The pressure in the reduction line is monitored and the reduction ends when the slope of the pressure becomes smaller than $0.3 \%$. In order to reduce the $\mathrm{CH}^{-}$output of our targets (see below), the excess $\mathrm{H}_{2}$ is pumped as soon as the reduction ends. The oven is brought down to room temperature and the trapped water is evacuated one sample at a time. The iron and graphite powder is pressed into the $1-\mathrm{mm}$ hole of the cathode. The cathode is kept under argon until it is measured.

\section{MEASUREMENTS ON INTERCALIBRATION AND BLANK SAMPLES}

Table 1 shows the results of our measurements on intercalibration samples.

Table 1 Measurements on intercalibration samples (1- $\sigma$ error of the mean).

\begin{tabular}{lcll}
\hline Sample type & Nr of targets & Value measured at ARTEMIS & Consensus value \\
\hline FIRI C & 6 & $18,316 \pm 64 \mathrm{BP}$ & $18,183 \pm 13 \mathrm{BP}$ \\
IAEA C2 & 9 & $41.45 \pm 0.20 \mathrm{pMC}$ & $41.14 \pm 0.03 \mathrm{pMC}$ \\
FIRI E & 7 & $11,714 \pm 42 \mathrm{BP}$ & $11,780 \pm 7 \mathrm{BP}$ \\
FIRI G & 12 & $110.56 \pm 0.15 \mathrm{pMC}$ & $110.7 \pm 0.04 \mathrm{pMC}$ \\
FIRI H & 11 & $2231 \pm 13 \mathrm{BP}$ & $2238 \pm 6 \mathrm{BP}$ \\
FIRI I & 3 & $4472 \pm 16 \mathrm{BP}$ & $4485 \pm 5 \mathrm{BP}$ \\
IAEA C6 & 65 & $150.11 \pm 0.07 \mathrm{pMC}$ & $150.61 \pm 0.11 \mathrm{pMC}$ \\
\hline
\end{tabular}

Table 2 summarizes the results on our blanks since the last modification on our graphitization procedure.

Table 2 Measurements on blanks (1- $\sigma$ error of the mean).

\begin{tabular}{lrll}
\hline & & \multicolumn{2}{c}{ Values measured at ARTEMIS } \\
\cline { 3 - 4 } Sample type & Nr of targets & pMC & age BP \\
\hline Charcoal & 32 & $0.188 \pm 0.015$ & $51,200 \pm 650$ \\
Tridacna shell & 5 & $0.121 \pm 0.012$ & $54,110 \pm 819$ \\
IAEA C1 & 15 & $0.109 \pm 0.006$ & $54,940 \pm 439$ \\
\hline
\end{tabular}

\section{THE FIRST YEARS OF OPERATION}

Becoming familiar with the system has been fairly easy. After some initial problems with the ion source sample changer, it has been performing reliably. A gradual loss of terminal voltage stability was observed after a little more than a year of operation. NEC traced the problem to a deterioration of the conducting properties of the pick-off pulleys in the terminal resulting in an abnormal wear of the drive sheaves due to incorrect down-charging of the chains. The pick-off pulleys have been temporarily eliminated and the charging system works on up-charging only. The stability of the terminal voltage is $\pm 0.6 \mathrm{kV}$ at $2.6 \mathrm{MV}$.

During the first year of operation, a problem with the reproducibility of our standards appeared correlated with $\mathrm{CH}^{-}$output determined from the ${ }^{13} \mathrm{C}^{-/ 12} \mathrm{C}^{-}$ratio (see Figure 3). The higher the $\mathrm{CH}^{-}$, the 
lower the ${ }^{14} \mathrm{C} /{ }^{12} \mathrm{C}$ ratios (as much as $4 \%$ ). The ${ }^{13} \mathrm{C}^{3+} / 12 \mathrm{C}^{3+}$ ratios, however, remain constant. This seems to exclude the possibility of not measuring correctly the high-energy ${ }^{13} \mathrm{C}^{3+}$ and ${ }^{12} \mathrm{C}^{3+}$ currents in the offset Faraday cups. The picture of the diaphragms in the front of these cups (Figure 4) shows the impact of the ${ }^{13} \mathrm{C}^{3+}$ and ${ }^{12} \mathrm{C}^{3+}$ beams coming from the corresponding $\mathrm{CH}^{-}$molecules. Since the spot on the ${ }^{12} \mathrm{C}^{3+}$ is rather close to the aperture, we reduced the size of the diaphragms. This did not eliminate the problem. We checked different parameters-stripper pressure, ramps in the bouncing system, dead-time correction-to see if we could eliminate or reduce this anticorrelation without success.

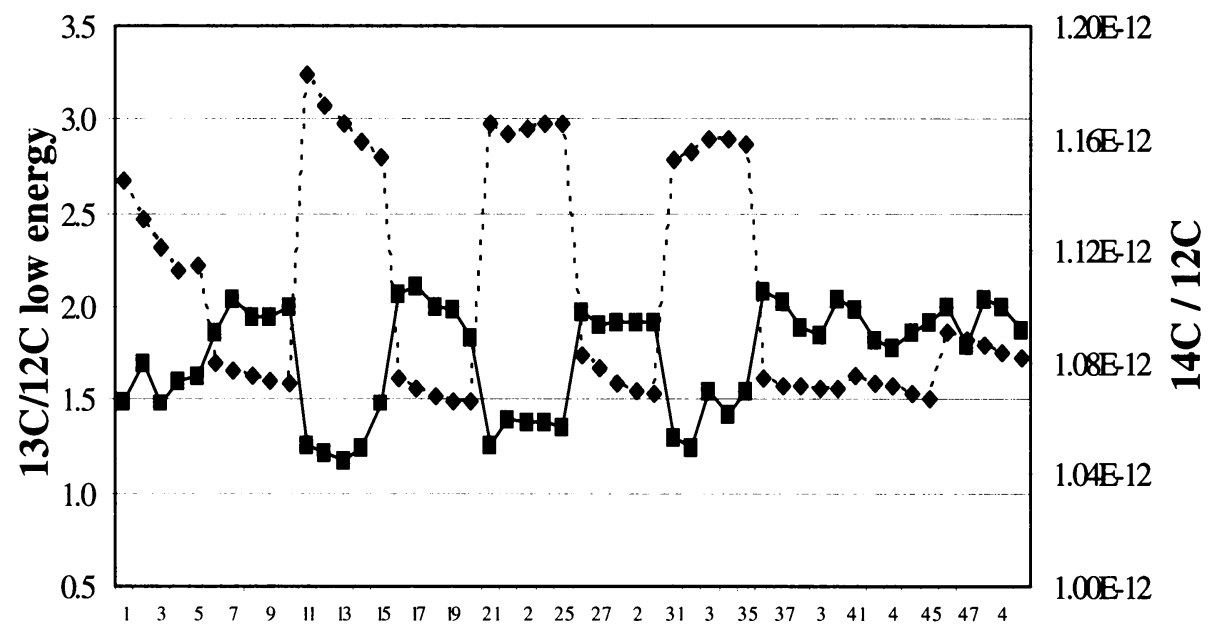

Figure 3 The ${ }^{14} \mathrm{C} /{ }^{12} \mathrm{C}$ ratio (squares) is anticorrelated to the ${ }^{13} \mathrm{C}^{-} /{ }^{12} \mathrm{C}^{-}$ratio (diamonds), which reflects the $\mathrm{CH}^{-}$output of the target.
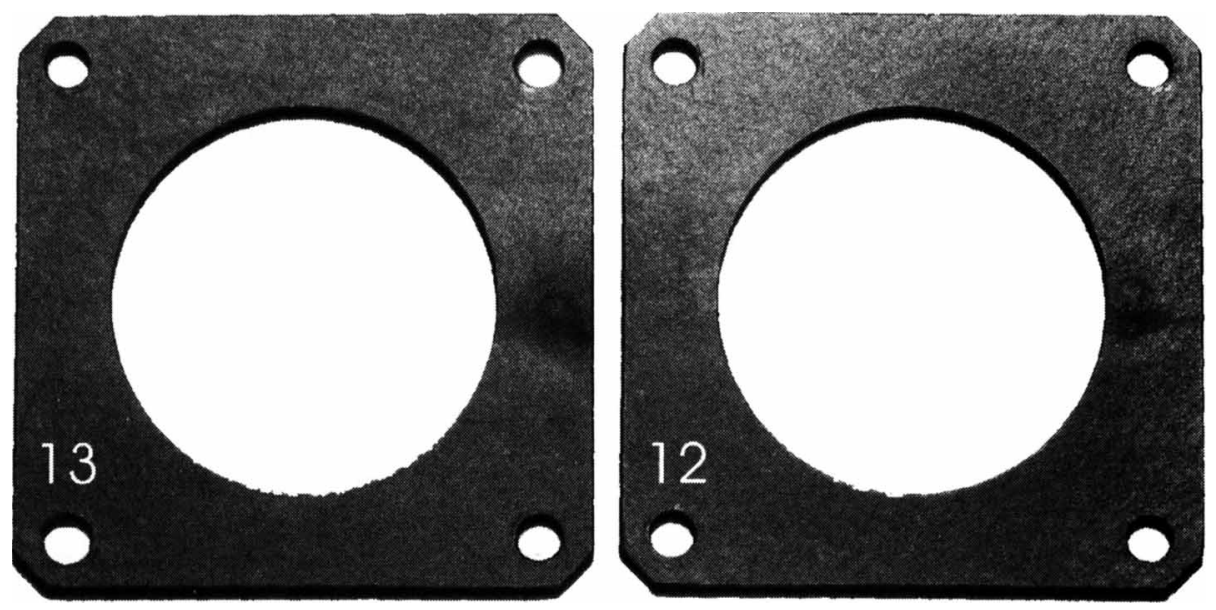

Figure 4 Diaphragms in front of the ${ }^{13} \mathrm{C}^{3+}$ and the ${ }^{12} \mathrm{C}^{3+}$ offset Faraday cups after the high-energy magnet showing the impact of the ${ }^{13} \mathrm{C}^{3+}$ and ${ }^{12} \mathrm{C}^{3+}$ beams coming from the corresponding $\mathrm{CH}^{-}$molecules.

The problem turned out to be related to the treatment of targets after pressing. The targets were put into vials and pumped for a certain time, sometimes overnight. Then, the vials were filled with argon 
and sealed. Cathodes treated that way did not behave in the same way as cathodes that had not been pumped and re-exposed to air upon installation on the wheel, as shown in Figure 5. The pumped cathodes show steeply varying $\mathrm{CH}^{-}$outputs as the runs proceed, while the $\mathrm{CH}^{-}$output of cathodes that were not pumped show rather flat profiles. The decrease of ${ }^{14} \mathrm{C} /{ }^{12} \mathrm{C}$ ratios could be correlated to the length of time the cathodes were pumped (Figure 6). We have not yet been able to explain what happens to the cathode material that can induce this change in ${ }^{14} \mathrm{C} /{ }^{12} \mathrm{C}$ ratio. Contamination with dead carbon from the pump does not seem like a reasonable explanation since several tens of $\mu \mathrm{g}$ of dead carbon would be needed, which seems a lot for an oil-free pump. Another possibility could be a modification of the target material induced by exposing the target back to air when installing the target in the ion source.

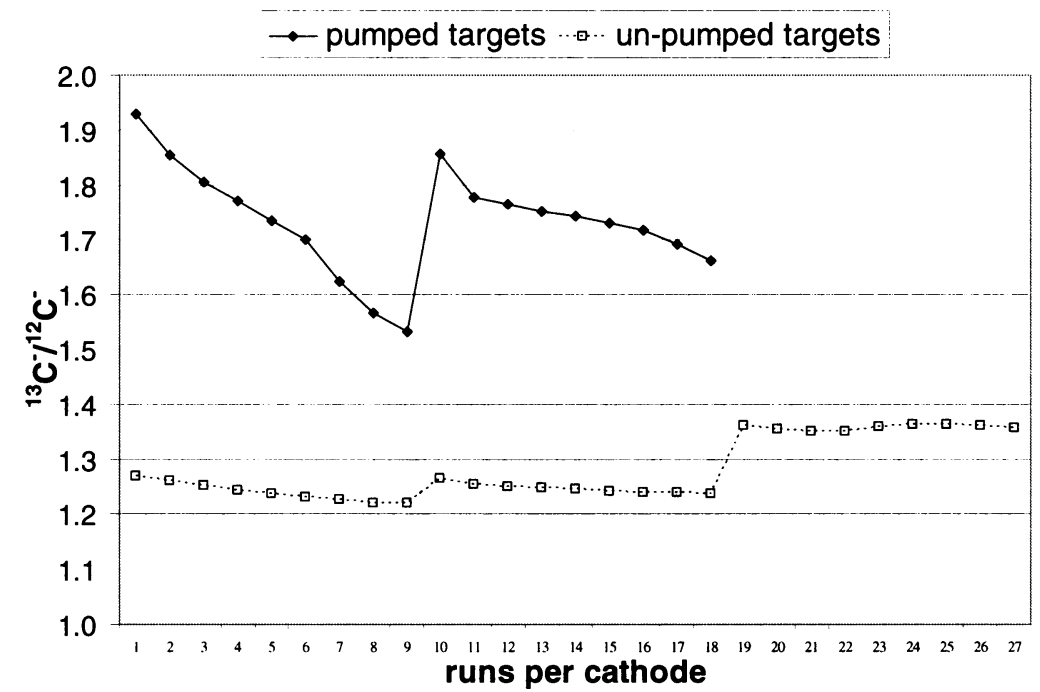

Figure 5 Variations of $\mathrm{CH}^{-}$output during the runs for pumped (diamonds) and unpumped (squares) targets.

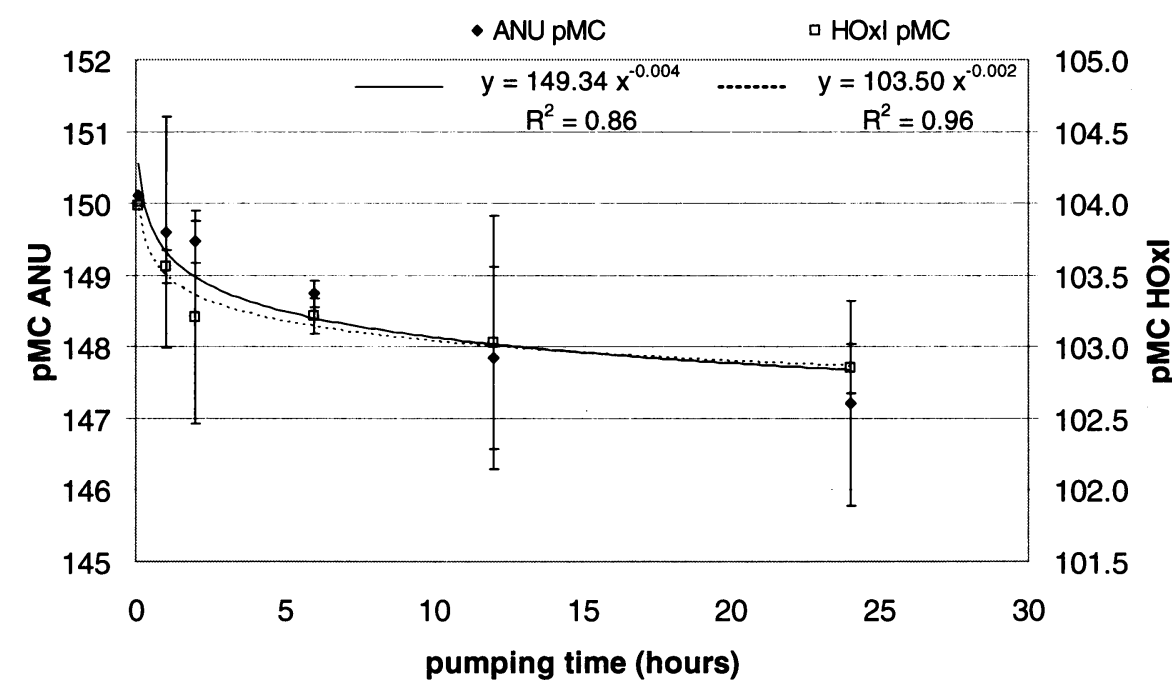

Figure 6 Average measured pMC decreases with the pumping time on the targets both for ANU (diamonds) and HOXI (squares) targets. 
The other problem related to the $\mathrm{CH}^{-}$output of our targets concerns the background samples. Blanks (C1B) from a large volume of $\mathrm{CO}_{2}$ made from $\mathrm{C} 1$ IAEA were graphitized and measured. Figure 7 shows a clear correlation $\left(R^{2} 0.53\right)$ between the pMC of these samples and their $\mathrm{CH}^{-}$output.

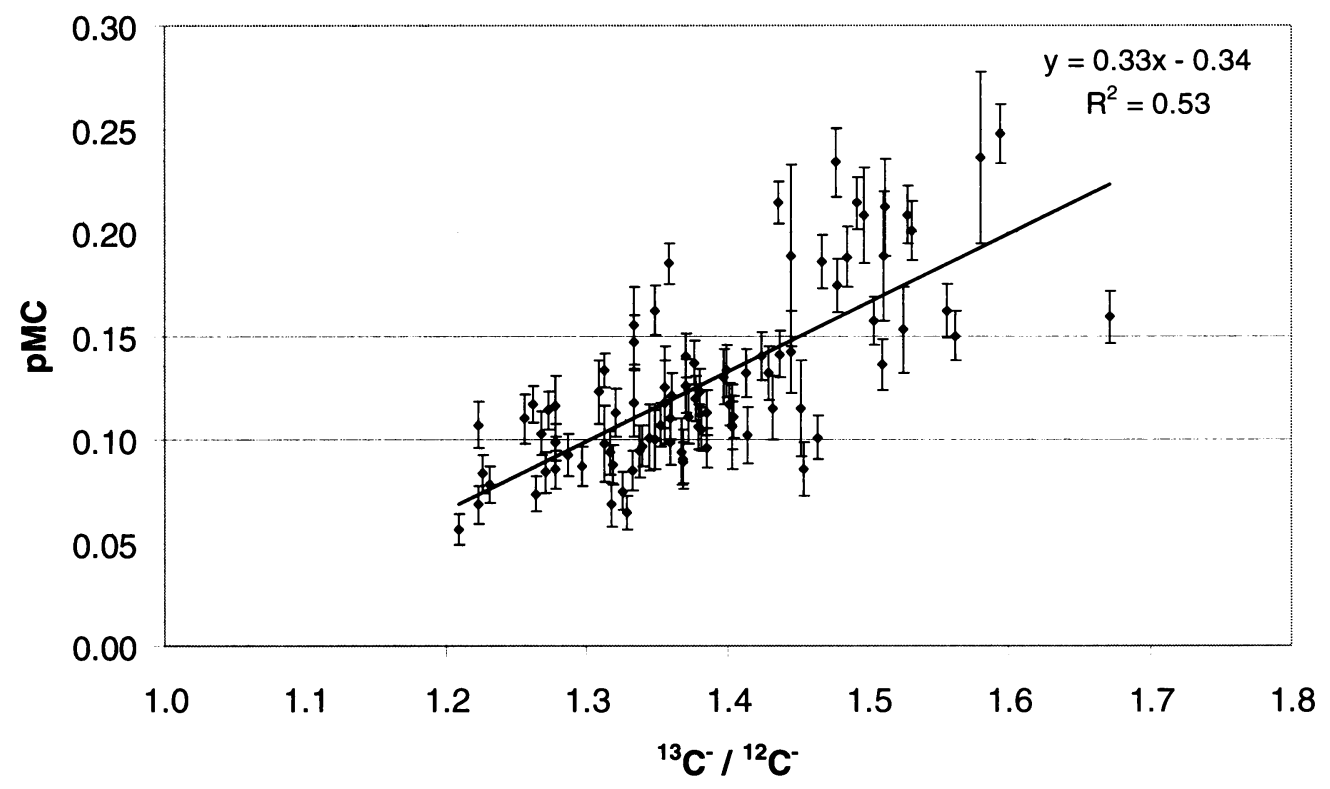

Figure 7 Correlation between the pMC values of targets graphitized from a large volume of $\mathrm{CO}_{2}$ made from IAEA C1 and their $\mathrm{CH}^{-}$output.

In order to investigate the background in the detector, we used 2 cathodes of $\mathrm{C} 1 \mathrm{~B}$ with low and high $\mathrm{CH}^{-}$output along with $2 \mathrm{HOXI}$ cathodes with low and high $\mathrm{CH}^{-}$output. We scanned the high-energy magnet BM03-1 between the 2 following values:

- ${ }^{13} \mathrm{C}^{3+} \mathrm{B}=12,350$ Gauss

- ${ }^{14} \mathrm{C}^{3+} \mathrm{B}=12,816$ Gauss

We counted during 100 seconds (Figure 8). Figure 8a shows the particles detected (in counts per second) per $\mu \mathrm{A}$ of injected ${ }^{12} \mathrm{C}^{-}$without varying the ECA field tuned for ${ }^{14} \mathrm{C}^{3+}$. The second plot (Figure $8 \mathrm{~b})$ shows the particles detected (in counts per second) per $\mu \mathrm{A}$ of injected ${ }^{12} \mathrm{C}^{-}$with the ECA field adjusted to the rigidity according to the following expression for each value of the magnetic field:

$$
\frac{E C A_{2}}{E C A_{1}}=\left(\frac{\text { rigidity }}{\text { rigidity }}\right)^{2}
$$

Both graphs show that the higher the $\mathrm{CH}^{-}$output of the target, the more particles reach the detector at all fields between mass 14 and 13. This background was not reduced by setting a voltage of the bouncing system for mass 14 (Southon et al. 2004). Charge exchange and diffusion on the residual gas along the rare isotope line could allow ${ }^{13} \mathrm{C}$ from ${ }^{13} \mathrm{CH}$ to enter the detector. NEC installed an additional turbomolecular pump under the ECA on the rare isotope line to reduce the pressure of the residual gas and thus reduce the probability of diffusion. 

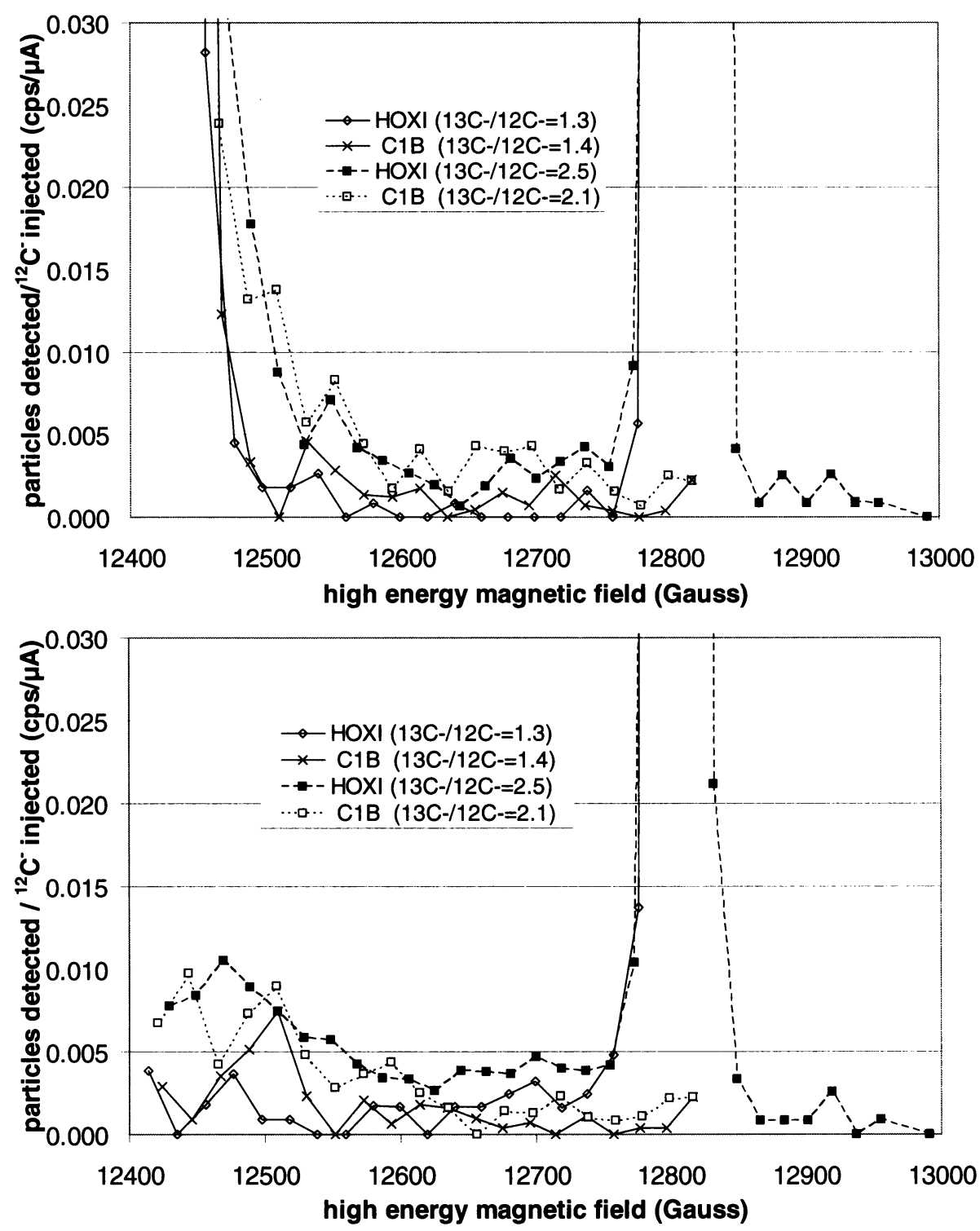

Figure 8 Scans of the high-energy magnetic field for cathodes with low (HOxI [diamonds] and C1B $[x])$ and high (HOxI [filled squares] and $\mathrm{C1B}$ [empty squares]) $\mathrm{CH}^{-}$output between $12,350 \mathrm{G}\left({ }^{13} \mathrm{C}^{3+}\right.$ ) and 12,816 G $\left({ }^{14} \mathrm{C}^{3+}\right)$ showing the particles detected (cps) per $\mu \mathrm{A}$ of injected ${ }^{12} \mathrm{C}^{-}:$a) with a fixed value for the ECA field resulting from the tuning for ${ }^{14} \mathrm{C}^{3+} ; \mathrm{b}$ ) with a value of the ECA field adjusted to the rigidity for each value of the magnetic field. The higher the $\mathrm{CH}^{-}$output of the target, the more particles reach the detector.

In order to be able to perform meaningful measurements, we changed our graphitization procedure as described above to lower the $\mathrm{CH}^{-}$output of our samples, which is now between 1.2 and 1.4.

\section{CONCLUSIONS}

Our AMS system Artemis is now operating routinely. Two phenomena appear when the $\mathrm{CH}^{-}$output of the targets is too high: 
1. Decrease of the ${ }^{14} \mathrm{C} /{ }^{12} \mathrm{C}$ ratio (up to about $4 \%$ for $\mathrm{HOxI}$ ): this might be due to some modification of the target that we do not yet understand, induced by exposing the target back to air after storage under argon. The targets are no longer pumped before storage under argon.

2. Unwanted counts in the detector: this was solved by changing both the reduction and storage processes so as to reduce the $\mathrm{CH}^{-}$output of our targets and adding a pump on the rare isotope line to reduce the charge exchange and diffusion of particles.

\section{ACKNOWLEDGMENTS}

We thank Martine Paterne and the Radiocarbon Group at LSCE (Gif sur Yvette, France) for their collaboration on sample preparation. We thank Jean-Jacques Poupeau and Jean-François Tannau for the construction of the sample preparation rigs. We thank James Ferry, Mark Sundquist, Roger Loger, Mike Stier, Richard Kitchen, and others at NEC for their help. We also thank Peter Steier, Walter Kutschera, and Alfred Priller at the VERA Laboratory in Vienna for discussions and measurements. We would also like to thank John Southon and Guaciara dos Santos of the University of California (Irvine, USA), Gordon Cook at NERC (Scotland), and Tanya Ertunç at SUERC (Scotland) for sending us some cathodes; and Mark Roberts at WHOI and Marie-Josée Nadeau at Kiel for measuring some samples. Last, but not the least, we want to thank Warren Beck at the University of Arizona (Tucson, USA) who sent us some samples and measured some of ours, and Stuart Freeman at SUERC for useful discussions.

This work was supported by CNRS, CEA, IRD, IRSN, and the Ministère de la Culture et de la Communication.

\section{REFERENCES}

Arnold M, Bard E, Maurice P, Duplessy JC. $1987 .{ }^{14} \mathrm{C}$ dating with the Gif-sur-Yvette Tandetron accelerator: status report. Nuclear Instruments and Methods in Physics Research B 29(1-2):120-3.

Hatté C, Poupeau J-J, Tannau J-F, Paterne M. 2003. Development of an automated system for preparation of organic samples. Radiocarbon 45(3):421-30.

Southon J, Santos G, Druffel-Rodriguez D, Druffel E, Trumbore S, Xu X, Griffin S, Ali S, Mazon M. 2004. The Keck Carbon Cycle AMS Laboratory, University of California, Irvine: initial operation and a background surprise. Radiocarbon 46(1):41-9.

Tisnérat-Laborde N, Poupeau J-J, Tannau J-F, Paterne M. 2001. Development of a semi-automated system for routine preparation of carbonate samples. Radiocarbon 43(2A):299-304.

Vogel JS, Southon JR, Nelson DE, Brown TA. 1984. Performance of catalytically condensed carbon for use in accelerator mass spectrometry. Nuclear Instruments and Methods in Physics Research B 5(2):289-93. 